\title{
Folding mechanism of canine milk lysozyme studied by circular dichroism and fluorescence spectroscopy
}

\author{
Masaharu Nakao ${ }^{a}$, Munehito Arai ${ }^{\mathrm{a}, 1}$, Takumi Koshiba ${ }^{\mathrm{b}, 2}$, Katsutoshi Nitta ${ }^{\mathrm{b}}$ and \\ Kunihiro Kuwajima ${ }^{\text {a,* }}$ \\ ${ }^{a}$ Department of Physics, Graduate School of Science, University of Tokyo, 7-3-1 Hongo, Bunkyo-ku, \\ Tokyo 113-0033, Japan \\ ${ }^{\mathrm{b}}$ Division of Biological Sciences, Graduate School of Science, Hokkaido University, N10 W8, Kita-ku, \\ Sapporo, Hokkaido 060-0810, Japan
}

\begin{abstract}
We have studied the guanidine hydrochloride-induced equilibrium unfolding and the kinetics of refolding of canine milk lysozyme by circular dichroism and fluorescence spectroscopy. The thermodynamic analysis of the equilibrium unfolding measured by circular dichroism and fluorescence has shown that unfolding is represented by a three-state mechanism and that the intermediate state of canine milk lysozyme is remarkably more stable than the intermediates observed in other lysozyme and $\alpha$-lactalbumin. In the kinetic refolding of this protein, there are at least two kinetic intermediates; a burst-phase intermediate accumulated within the dead time (4 ms) of the measurement and an intermediate that has been observed during the kinetics with a rate constant of $10-20 \mathrm{~s}^{-1}$ after the burst phase. This result is apparently in contrast with those previously observed in the kinetic refolding of $\alpha$-lactalbumin and equine lysozyme that show only the burst-phase intermediate. The relationship between the extraordinarily stable equilibrium molten globule and the kinetic folding intermediates will be discussed.
\end{abstract}

\section{Introduction}

In order to elucidate the mechanism of protein folding, kinetic analysis of refolding from a fully unfolded state and detection and characterization of the intermediates observed in the equilibrium and kinetic measurements are fundamental strategies [1,2]. The molten globule (MG) state is known to be a common physical state observed as an equilibrium intermediate of a number of globular proteins, and studies on various proteins have shown that the MG state is equivalent to a transient kinetic intermediate observed in the refolding process from the guanidine hydrochloride $(\mathrm{GdnHCl})$-induced unfolded state, indicating that the MG state is a general intermediate of protein folding [1,3-8].

Canine milk lysozyme is a $\mathrm{Ca}^{2+}$-binding lysozyme, containing 129 amino acid residues with a molecular weight of 14,600, and a crystallographic study has shown that the structure of canine milk lysozyme is very similar to those of other lysozyme and $\alpha$-lactalbumin [9]. The structure of lysozyme consists of

\footnotetext{
${ }^{1}$ Present address: Institute of Molecular and Cell Biology, National Institute of Advanced Industrial Science and Technology, 1-1-1 Higashi, Tsukuba, Ibaraki 305-8566, Japan.

${ }^{2}$ Present address: Division of Biology, MC 156-29, California Institute of Technology, 1200 E. California Blvd., Pasadena, CA 91125 , USA.

${ }^{*}$ Corresponding author. Dr. Kunihiro Kuwajima, Tel.: +81 35841 4128; Fax: +81 35841 4512; E-mail: kuwajima@ phys.s.utokyo.ac.jp.
} 
two domains, an $\alpha$-domain and a $\beta$-domain. The $\alpha$-domain mainly consists of four $\alpha$-helices, and the $\beta$-domain is formed by a series of loops and three anti-parallel $\beta$-strands.

An equilibrium thermal unfolding study has shown that canine milk lysozyme exhibits a MG-like intermediate that is more stable and more native-like in the structure than those of other lysozyme and $\alpha$-lactalbumin [9]. The MG states of other lysozyme and $\alpha$-lactalbumin observed in the equilibrium $\mathrm{GdnHCl}$-induced unfolding are known to be identical to the kinetic folding intermediates $[8,10]$. Thus, it is interesting to investigate whether or not such a more stable and more native-like MG intermediate as observed in the thermal unfolding is also observed in the GdnHCl-induced equilibrium unfolding of canine milk lysozyme and, if the intermediate is observed, whether or not there is any relationship between the intermediate and the kinetic folding intermediate. Because the folding intermediate of canine milk lysozyme is expected to be remarkably more stable and more native-like than the intermediates previously studied in other lysozyme and $\alpha$-lactalbumin, studies of the canine milk lysozyme intermediate may provide a key to understanding the folding mechanism of the proteins in the lysozyme and $\alpha$-lactalbumin family.

In this study, we have investigated the GdnHCl-induced equilibrium unfolding of canine milk lysozyme by circular dichroism (CD) and fluorescence spectroscopy and the kinetic refolding of the protein by a stopped-flow technique. It is shown that the equilibrium unfolding transition of the highly purified canine milk lysozyme is represented by a three-state mechanism that involves the MG-like intermediate and that there are at least two intermediates in the kinetic refolding of the protein that exhibit overshoot behavior observed by a time dependent ellipticity change at $222 \mathrm{~nm}$.

\section{Materials and methods}

\subsection{Reagents}

$\mathrm{GdnHCl}$ was of specially prepared reagent grade for biochemical use from Nacalai Tesque Inc. (Kyoto, Japan). Other chemicals were of guaranteed reagent grade. Concentration of $\mathrm{GdnHCl}$ was determined by an Atago 3T refractometer. The concentration of canine milk lysozyme was determined spectrophotometrically at $280 \mathrm{~nm}$ with an extinction coefficient of $E_{1 \%}^{1 \mathrm{~cm}}=23.2$ [12].

\subsection{Refolding and purification of canine milk lysozyme}

Canine milk lysozyme expressed as inclusion bodies in Escherichia coli (E. coli) cells was purified and refolded by the method of Koshiba et al. [13] with modification. The E. coli cells that contained canine milk lysozyme were lysed by sonication. The inclusion bodies were collected by centrifugation and washed. The washed inclusion bodies were suspended in $6 \mathrm{M} \mathrm{GdnHCl}$ that contains $50 \mathrm{mM}$ Tris- $\mathrm{HCl}$ (pH 8.0), $1 \mathrm{mM}$ EDTA, and 0.1 $\mathrm{M}$ dithiothreitol (DTT), and kept at room temperature overnight. The unsolubilized debris was removed by centrifugation, and the solubilized protein was partially purified by gel filtration on a column of Sephacryl S-100 equilibrated with the equilibration buffer ( $50 \mathrm{mM}$ Tris- $\mathrm{HCl}$ (pH 8.0), $1 \mathrm{mM}$ EDTA, and $6 \mathrm{M} \mathrm{GdnHCl}$ ). The fraction containing lysozyme was pooled and used for refolding.

The refolding of recombinant canine milk lysozyme that had been partially purified by gel filtration was carried out as follows. Denatured recombinant canine milk lysozyme was first diluted to 25 or $50 \mu \mathrm{M}$ using the equilibration buffer. A solution of thioredoxin $(50 \mu \mathrm{M})$ was prepared ten times as much volume as lysozyme solution. Half of them were incubated with $10 \mu \mathrm{M}$ DTT for 30 minutes to 
make reduced form of thioredoxin. The rest of them was oxygenated by bubbling $\mathrm{O}_{2}$ gas. To make the refolding solution, the same volume of the thioredoxin solutions of the reduced and oxidized forms were mixed, and $0.5 \mathrm{M} \mathrm{CaCl}_{2}$ was added to the solution so that the final concentration of $\mathrm{CaCl}_{2}$ was $1 \mathrm{mM}$. Finally, the denatured canine milk lysozyme solution was added immediately to the refolding solution, and mixed using magnetic stirrer so that the volume ratio of the lysozyme solution to the refolding buffer was $1: 10$. The reaction mixture was incubated for 4 days at $25^{\circ} \mathrm{C}$.

The reaction mixture of the lysozyme-thioredoxin solution was dialyzed against $20 \mathrm{mM}$ Tris buffer (pH 8.0) that contained $1 \mathrm{mM} \mathrm{CaCl} 2$, and applied to an SP-sepharose FF column equilibrated with $20 \mathrm{mM}$ Tris buffer ( $\mathrm{pH} 8.0$ ) that contained $20 \mathrm{mM} \mathrm{CaCl}_{2}$. Refolded recombinant canine milk lysozyme was eluted from the column by a linear gradient of $\mathrm{NaCl}$ from 0 to $0.1 \mathrm{M}$ (4 column volumes). A reversedphase HPLC analysis indicated that the protein purified by SP-sepharose still contained a contaminant $(\sim 10 \%)$ that was about 19-Da larger in mass as estimated by mass spectrometry. The contaminant was thus separated by the HPLC as follows. The fraction containing lysozyme was collected and applied to a HPLC Octadecyl 4-PW column $(5.5 \times 20 \mathrm{~cm}$; Tosoh; Japan) at room temperature with a gradient of acetonitrile from 32 to $37 \%$ in 125 minutes using $0.1 \%$ TFA in distilled water and $0.07 \%$ TFA in acetonitrile. Each peak was collected individually. To remove acetonitrile and precipitate the refolded protein, $\left(\mathrm{NH}_{4}\right)_{2} \mathrm{SO}_{4}$ was added to the solutions. The solution was centrifuged at $20,000 \mathrm{~g}$ for 60 minutes at $4{ }^{\circ} \mathrm{C}$, and the precipitate and the aqueous phase that contained the purified refolded protein were collected and dialyzed against distilled water.

\subsection{Equilibrium experiments}

GdnHCl-induced equilibrium unfolding transitions of canine milk lysozyme were measured by CD and fluorescence spectroscopy. Samples were prepared in $50 \mathrm{mM}$ sodium cacodylate buffer ( $\mathrm{pH}$ 7.0) that contained $50 \mathrm{mM} \mathrm{NaCl}, 1 \mathrm{mM} \mathrm{CaCl}_{2}$, and indicated concentrations of $\mathrm{GdnHCl}$. Equilibrium CD spectra and unfolding transition were measured using a Jasco J-720 spectropolarimeter. The path lengths of optical cuvettes were $2.0 \mathrm{~mm}$ and $10.0 \mathrm{~mm}$ for the far-ultra violet (UV) CD and the near-UV CD measurements, respectively. The temperature of the cuvette was controlled by circulating water at $25^{\circ} \mathrm{C}$. The concentration of lysozyme was 5.5-5.6 $\mu \mathrm{M}$. The GdnHCl-induced equilibrium unfolding transition curves of canine milk lysozyme were measured at $222 \mathrm{~nm}$ and $295 \mathrm{~nm}$ for the far- and near-UV CD ellipticity, respectively. Intrinsic fluorescence spectra measurements were performed using a Jasco FP-777 spectrofluorometer (Tokyo, Japan). The exciting wavelength was $295 \mathrm{~nm}$, and slits for excitation and emission were $1.5 \mathrm{~nm}$ and $3 \mathrm{~nm}$, respectively. Fluorescence measurements of equilibrium unfolding transitions were carried out using an SX.18MV stopped-flow spectrometer from Applied Photophysics (Leatherhead, UK). After excitation at $295 \mathrm{~nm}$, we measured the total fluorescence emission above $370 \mathrm{~nm}$ using a cut-off filter (SC-37, Fuji Photo Film Co. Ltd., Japan) or that around $350 \mathrm{~nm}$ using a band-pass filter (U-350, Hoya Co., Japan). The temperature in the apparatus was maintained at $25^{\circ} \mathrm{C}$ using circulating water. The concentration of lysozyme was $0.68 \mu \mathrm{M}$ in all equilibrium fluorescence measurements.

\subsection{Kinetic experiments}

The protein solution was prepared in $50 \mathrm{mM}$ sodium cacodylate buffer $(\mathrm{pH} 7.0)$ that contained $50 \mathrm{mM}$ $\mathrm{NaCl}, 1 \mathrm{mM} \mathrm{CaCl}$, and $7 \mathrm{M} \mathrm{GdnHCl}$. The refolding buffer contained $50 \mathrm{mM}$ sodium cacodylate (pH 7.0), $50 \mathrm{mM} \mathrm{NaCl}$ and $1 \mathrm{mM} \mathrm{CaCl}_{2}$. The refolding reaction was initiated by mixing the protein 
solution with the refolding buffer; the mixing ratio of the protein solution and refolding buffer was $1: 10.4$. The final concentration of $\mathrm{GdnHCl}$ was $0.61 \mathrm{M}$. The temperature was controlled by circulating water at $25^{\circ} \mathrm{C}$ [8]. Kinetic far UV CD measurement was carried out using a stopped-flow apparatus (specially designed and constructed by Unisoku, Japan) installed in the cell compartment of the Jasco J-720 spectropolarimeter and had a deadtime of $25 \mathrm{~ms}$ [8]. An optical cell with a $3.9 \mathrm{~mm}$ optical path length was used. The resultant kinetics was observed at $222 \mathrm{~nm}$. Stopped-flow fluorescence measurements were performed using an SX.18MV stopped-flow spectrometer (Applied Photophysics; Leatherhead, UK). The excitation wavelength was $295 \mathrm{~nm}$, and the emission light through a $350 \mathrm{~nm}$ bandpass filter (U-350, Hoya Co., Japan) was observed. The deadtime of the measurements was $4 \mathrm{~ms}$.

\section{Results}

\subsection{Equilibrium measurements}

Figure 1 shows CD spectra of canine milk lysozyme (holo form) at $\mathrm{pH} 7.0$ and $25^{\circ} \mathrm{C}$. The near-UV CD spectrum shows characteristic positive Cotton effects at $289 \mathrm{~nm}$ and $295 \mathrm{~nm}$, and a trough at $292 \mathrm{~nm}$. It indicates the presence of specific rigid packing interactions of aromatic side chains. The far-UV CD spectrum shows large negative ellipticity from $215 \mathrm{~nm}$ to $230 \mathrm{~nm}$, indicating the presence of $\alpha$-helices. These characteristics of native $\mathrm{CD}$ spectra are lost in concentrated $\mathrm{GdnHCl}(7.87 \mathrm{M})$, where lysozyme is fully unfolded [13,14].

Figure 2 shows the $\mathrm{GdnHCl}$ concentration dependence of the fluorescence emission spectrum of canine milk lysozyme. Because the excitation wavelength is $295 \mathrm{~nm}$, the spectrum change is expected to reflect the environmental change of tryptophan residues present in canine milk lysozyme. The fluorescence spectrum has a maximum at $340 \mathrm{~nm}$ in the absence of $\mathrm{GdnHCl}$, and changes in a two-stage manner upon increasing $\mathrm{GdnHCl}$ concentration: (1) decreasing the fluorescence intensity, and (2) showing a red shift and an increase of the intensity.

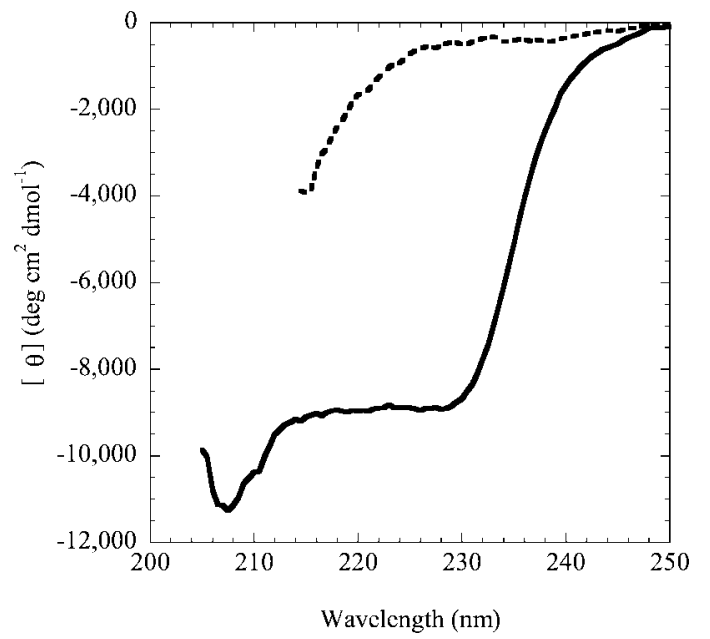

(a)

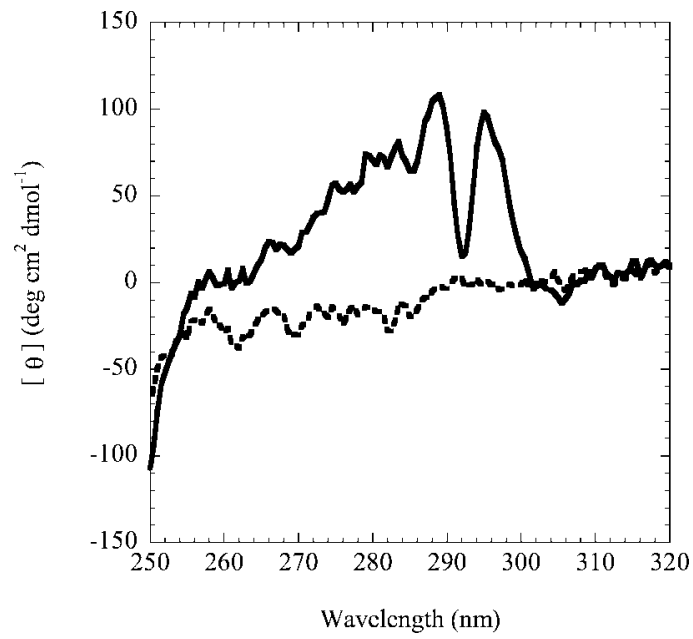

(b)

Fig. 1. CD spectra of holo canine milk lysozyme in (a) far and (b) near-UV region. The spectra of lysozyme in the $\mathrm{N}(0 \mathrm{M}$ $\mathrm{GdnHCl}$; continuous line) and $\mathrm{U}$ states $(7.87 \mathrm{M} \mathrm{GdnHCl}$; broken line) are shown. All solutions contain $50 \mathrm{mM}$ sodium cacodylate (pH 7.0), $50 \mathrm{mM} \mathrm{NaCl}$ and $1 \mathrm{mM} \mathrm{CaCl}_{2}$. 


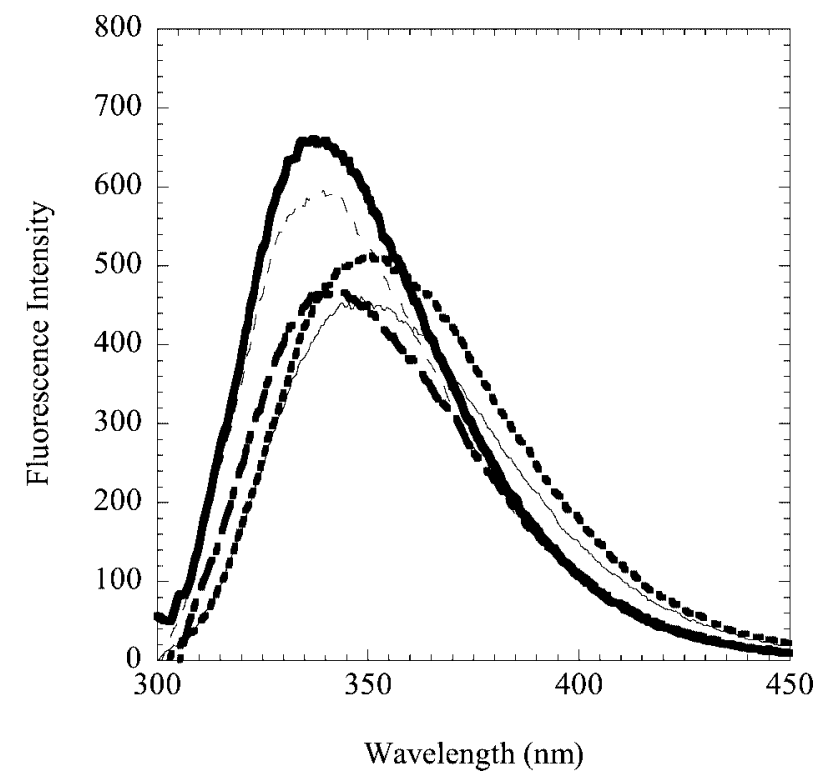

Fig. 2. Fluorescence spectra of holo canine milk lysozyme. The spectra of lysozyme in $0 \mathrm{M}$ (thick solid line), $2 \mathrm{M}$ (thin dashed line), $4 \mathrm{M}$ (thick dashed and dotted line), $6 \mathrm{M}$ (thin solid line) and $8 \mathrm{M} \mathrm{GdnHCl}$ (thick dotted line) are shown. All solutions contain $50 \mathrm{mM}$ sodium cacodylate (pH 7.0), $50 \mathrm{mM} \mathrm{NaCl}$ and $1 \mathrm{mM} \mathrm{CaCl}_{2}$.

GdnHCl-induced equilibrium unfolding transition curves of holo canine milk lysozyme measured by $\mathrm{CD}$ and fluorescence are shown in Figs 3 and 4, respectively. The transition curves measured by CD ellipticity at $222 \mathrm{~nm}$ and fluorescence emission above $370 \mathrm{~nm}$ apparently show a single-step transition. However, the curves measured by CD at $295 \mathrm{~nm}$ and fluorescence intensity around $350 \mathrm{~nm}$ indicate the presence of two transitions. To interpret the transition curves shown in Figs 3 and 4, we applied a threestate model to the unfolding transition as follows [10]. We first assume the unfolding of lysozyme to be expressed by the following formula:

$$
\mathrm{N} \rightleftharpoons \mathrm{I} \rightleftharpoons \mathrm{U}
$$

where $\mathrm{N}$, I, and $\mathrm{U}$ represent the native, the intermediate, and the unfolded states, respectively. We define the equilibrium constants between $\mathrm{N}$ and I and between $\mathrm{N}$ and $\mathrm{U}$ as $K_{\mathrm{NI}}\left(=f_{\mathrm{I}}(c) / f_{\mathrm{N}}(c)\right)$ and $K_{\mathrm{NU}}(=$ $\left.f_{\mathrm{U}}(c) / f_{\mathrm{N}}(c)\right)$, respectively; where $f_{\mathrm{N}}(c), f_{\mathrm{I}}(c)$ and $f_{\mathrm{U}}(c)$ represent the fraction of the three states at a $\mathrm{GdnHCl}$ concentration of $c\left(f_{\mathrm{N}}+f_{\mathrm{I}}+f_{\mathrm{U}}=1\right)$.

The apparent ellipticity or fluorescence intensity of the protein observed in the measurements is the sum of the contributions from the three states as

$$
A_{\mathrm{obs}}(c)=A_{\mathrm{N}} f_{\mathrm{N}}(c)+A_{\mathrm{I}} f_{\mathrm{I}}(c)+A_{\mathrm{U}} f_{\mathrm{U}}(c),
$$

where $A_{\mathrm{N}}, A_{\mathrm{I}}$ and $A_{\mathrm{U}}$ are the values of the pure $\mathrm{N}, \mathrm{I}$ and $\mathrm{U}$ states, respectively. The fraction of every state can be related to the equilibrium constants, $K_{\mathrm{NI}}$ and $K_{\mathrm{NU}}$, and therefore, the corresponding free energy changes, $\Delta G_{\mathrm{NI}}$ and $\Delta G_{\mathrm{NU}}$, as: 


$$
\begin{aligned}
& f_{\mathrm{N}}=\frac{1}{1+K_{\mathrm{NI}}+K_{\mathrm{NU}}}=\frac{1}{1+\exp \left(-\Delta G_{\mathrm{NI}} / R T\right)+\exp \left(-\Delta G_{\mathrm{NU}} / R T\right)}, \\
& f_{\mathrm{I}}=\frac{K_{\mathrm{NI}}}{1+K_{\mathrm{NI}}+K_{\mathrm{NU}}}=\frac{\exp \left(-\Delta G_{\mathrm{NI}} / R T\right)}{1+\exp \left(-\Delta G_{\mathrm{NI}} / R T\right)+\exp \left(-\Delta G_{\mathrm{NU}} / R T\right)}, \\
& f_{\mathrm{U}}=\frac{K_{\mathrm{NU}}}{1+K_{\mathrm{NI}}+K_{\mathrm{NU}}}=\frac{\exp \left(-\Delta G_{\mathrm{NU}} / R T\right)}{1+\exp \left(-\Delta G_{\mathrm{NI}} / R T\right)+\exp \left(-\Delta G_{\mathrm{NU}} / R T\right)},
\end{aligned}
$$

where $R$ and $T$ are the gas constant and the absolute temperature, respectively. In general, we assume that the free energy of unfolding varies approximately linearly with $c$, so that:

$$
\begin{aligned}
& \Delta G_{\mathrm{NI}}=\Delta G_{\mathrm{NI}}^{\mathrm{H}_{2} \mathrm{O}}-m_{\mathrm{NI}} c, \\
& \Delta G_{\mathrm{NU}}=\Delta G_{\mathrm{NU}}^{\mathrm{H}_{2} \mathrm{O}}-m_{\mathrm{NU}} c,
\end{aligned}
$$

where $\Delta G_{\mathrm{NI}}^{\mathrm{H}_{2} \mathrm{O}}$ and $\Delta G_{\mathrm{NU}}^{\mathrm{H}_{2} \mathrm{O}}$ are the $\Delta G_{\mathrm{NI}}$ and $\Delta G_{\mathrm{NU}}$ at $0 \mathrm{M} \mathrm{GdnHCl}$, respectively, and $m_{\mathrm{NI}}$ and $m_{\mathrm{IU}}$ represent the cooperativity indexes of the transitions. From Eq. (1) to (3), $A_{\mathrm{obs}}(c)$ is given by:

$$
A_{\mathrm{obs}}(c)=\frac{A_{\mathrm{N}}+A_{\mathrm{I}} \exp \left[-\left(\Delta G_{\mathrm{NI}}^{\mathrm{H}_{2} \mathrm{O}}-m_{\mathrm{NI}} c\right) / R T\right]+A_{\mathrm{U}} \exp \left[-\left(\Delta G_{\mathrm{NU}}^{\mathrm{H}_{2} \mathrm{O}}-m_{\mathrm{NU}} c\right) / R T\right]}{1+\exp \left[-\left(\Delta G_{\mathrm{NI}}^{\mathrm{H}_{2} \mathrm{O}}-m_{\mathrm{NI}} c\right) / R T\right]+\exp \left[-\left(\Delta G_{\mathrm{NU}}^{\mathrm{H}_{2} \mathrm{O}}-m_{\mathrm{NU}} c\right) / R T\right]} .
$$

Here, we assume that $A_{\mathrm{N}}, A_{\mathrm{I}}$, and $A_{\mathrm{U}}$ have the linear dependence on $c$, as $A_{\mathrm{N}}=a_{1}+a_{2} c, A_{\mathrm{I}}=a_{3}+a_{4} c$ and $A_{\mathrm{U}}=a_{5}+a_{6} c$.

The data of Figs 3 and 4 were analyzed on the basis of Eq. (4) by the method of non-linear least squares. In this analysis, we performed the global fitting; the four transition curves were fitted simultaneously. The

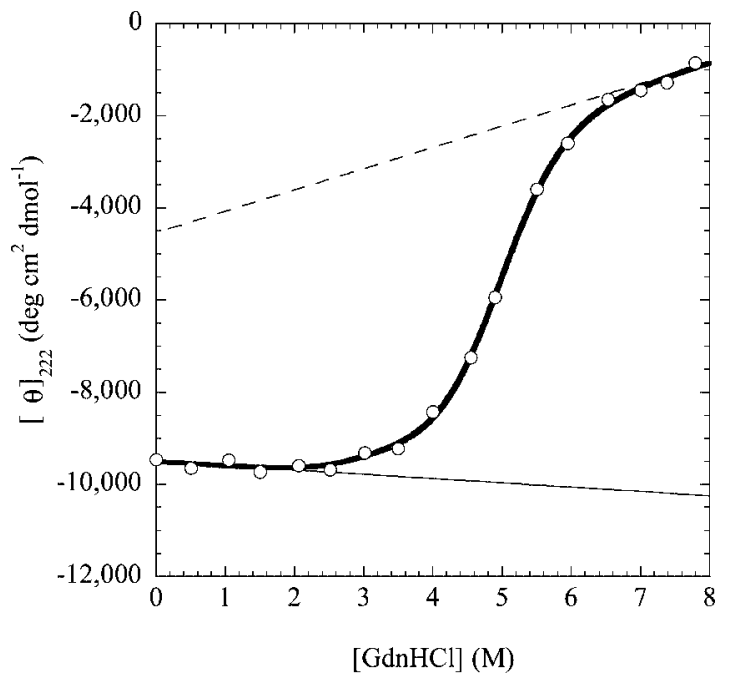

(a)

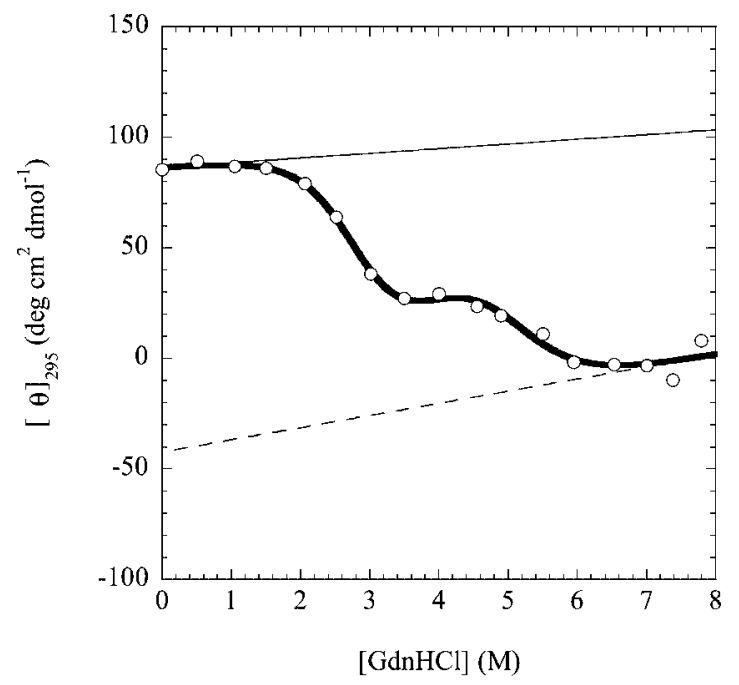

(b)

Fig. 3. Equilibrium unfolding transition curves of canine milk lysozyme measured by ellipticity at (a) $222 \mathrm{~nm}$, and (b) $295 \mathrm{~nm}$. Open symbols represent the data at each concentration of $\mathrm{GdnHCl}$. Thick continuous lines are the unfolding transition curves calculated by global fitting (see Results). The baselines of the $\mathrm{U}$ and $\mathrm{N}$ states are also shown. 


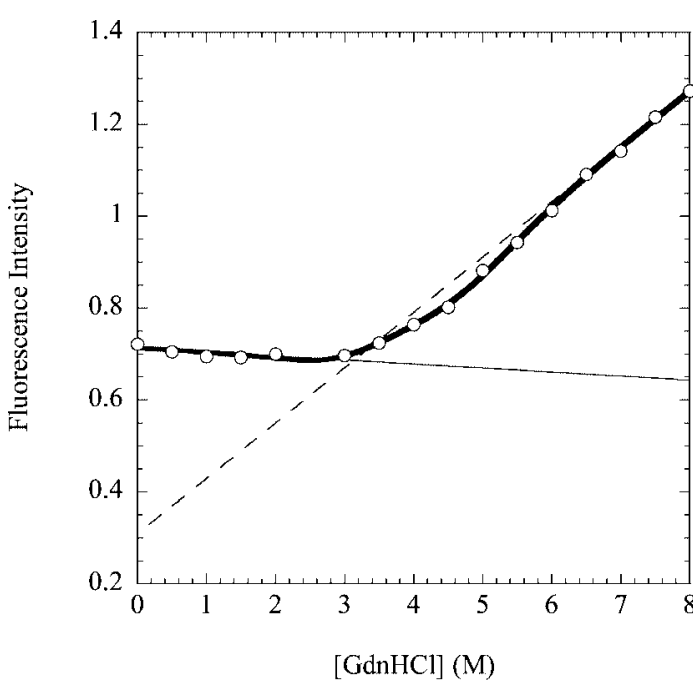

(a)

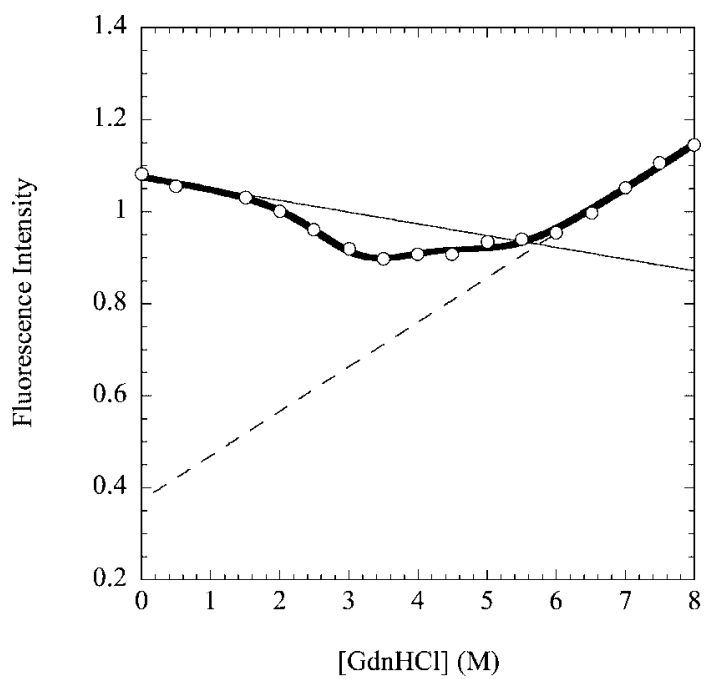

(b)

Fig. 4. Equilibrium unfolding transition curves of canine milk lysozyme measured by fluorescence emission (a) above $370 \mathrm{~nm}$ using a cut-off filter (SC-37, Fuji Photo Film Co. Ltd., Japan) and (b) that around $350 \mathrm{~nm}$ using a band-pass filter (U-350, Hoya Co., Japan). Open symbols represent the data at each concentration of $\mathrm{GdnHCl}$. Thick continuous lines are the unfolding transition curves calculated by global fitting (see Results). The baselines of the $\mathrm{U}$ and $\mathrm{N}$ states are also shown.

Table 1

Equilibrium unfolding parameters of holo lysozyme and $\alpha$-lactalbumin $\left(25^{\circ} \mathrm{C}\right)$

\begin{tabular}{ccccc}
\hline & $\begin{array}{c}\text { Canine } \\
\text { lysozyme } \\
(\mathrm{pH} \mathrm{7.0})\end{array}$ & $\begin{array}{c}\text { Equine } \\
\text { lysozyme } \\
(\mathrm{pH} \mathrm{7.0})\end{array}$ & $\begin{array}{c}\text { Bovine } \alpha- \\
\text { lactalbumin }^{(2)} \\
(\mathrm{pH} \mathrm{7.0)}\end{array}$ & $\begin{array}{c}\text { Canine } \\
\text { lysozyme }^{(3)} \\
(\mathrm{pH} \mathrm{4.5)}\end{array}$ \\
\hline$\Delta G_{\mathrm{NI}}^{\mathrm{H}_{2} \mathrm{O}}\left(\mathrm{kcal} \mathrm{mol}^{-1}\right)$ & $4.41 \pm 0.26$ & $5.64 \pm 0.32$ & 5.80 & 2.84 \\
$m_{\mathrm{NI}}\left(\mathrm{kcal} \mathrm{mol}^{-1} \mathrm{M}^{-1}\right)$ & $1.52 \pm 0.11$ & $2.00 \pm 0.11$ & 1.64 & 2.43 \\
$\Delta G_{\mathrm{IU}}^{\mathrm{H}_{2} \mathrm{O}}\left(\mathrm{kcal} \mathrm{mol}^{-1}\right)$ & $6.20 \pm 0.41$ & $4.33 \pm 0.59$ & 1.42 & 5.27 \\
$m_{\mathrm{IU}}\left(\mathrm{kcal} \mathrm{mol}^{-1} \mathrm{M}^{-1}\right)$ & $1.26 \pm 0.16$ & $1.13 \pm 0.19$ & 0.75 & 1.00 \\
$\Delta G_{\mathrm{NU}}^{\mathrm{H}_{2} \mathrm{O}}\left(\mathrm{kcal} \mathrm{mol}^{-1}\right)$ & $10.61 \pm 0.31$ & $9.97 \pm 0.49$ & 7.22 & 8.11 \\
$m_{\mathrm{NU}}\left(\mathrm{kcal} \mathrm{mol}^{-1} \mathrm{M}^{-1}\right)$ & $2.78 \pm 0.11$ & $3.13 \pm 0.15$ & 2.39 & 3.43 \\
\hline
\end{tabular}

\footnotetext{
${ }^{(1)}$ Mizuguchi et al. [10].

${ }^{(2)}$ Ikeguchi et al. [15].

${ }^{(3)}$ Kobashigawa et al. [14].
}

fitting variables were $\Delta G_{\mathrm{NI}}^{\mathrm{H}_{2} \mathrm{O}}, \Delta G_{\mathrm{NU}}^{\mathrm{H}_{2} \mathrm{O}}, m_{\mathrm{NI}}$ and $m_{\mathrm{NU}}$, and four sets of $a_{1}$ to $a_{6}$ for the transition curves. The obtained values of parameters are given in Table 1.

The continuous lines in Figs 3 and 4 are theoretical curves drawn with the parameter values shown in Table 1. The theoretical curves show good agreement with the experimental data, indicating that the three-state analysis is valid for the data, and only the three states, the N, I, and U states, are sufficient and no other state is required for interpreting the unfolding transition of canine milk lysozyme. 


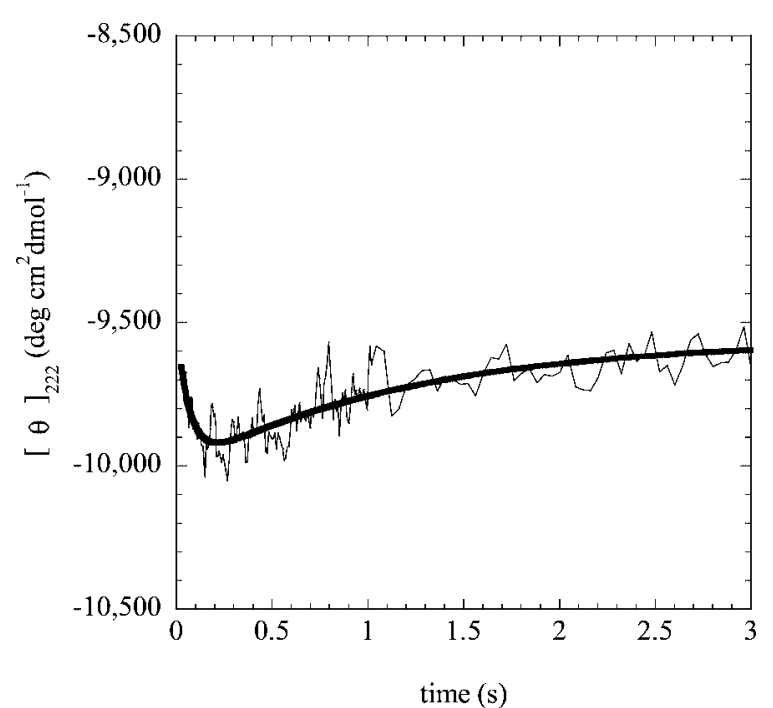

(a)

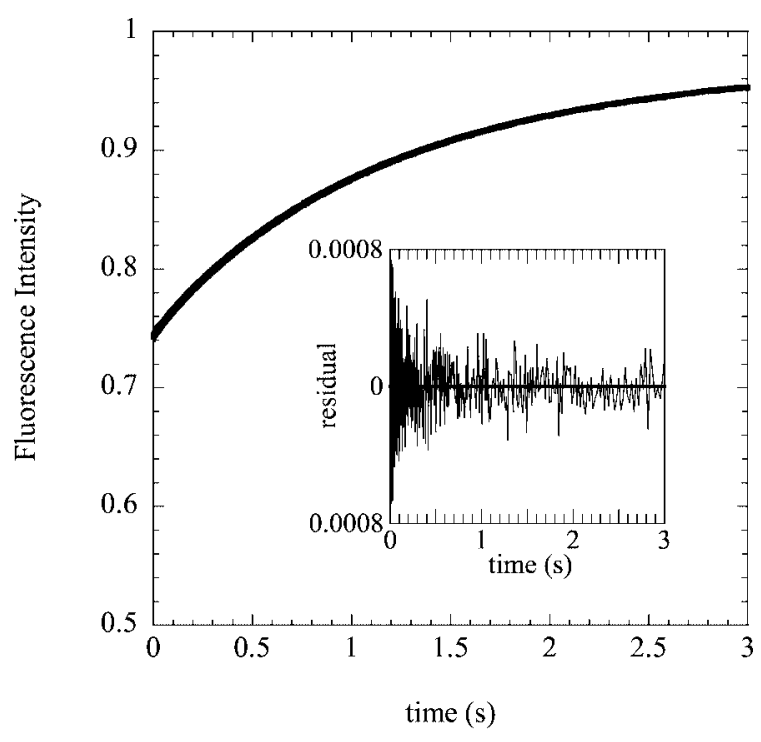

(b)

Fig. 5. Kinetic refolding curves of holo canine milk lysozyme measured by (a) far UV CD at $222 \mathrm{~nm}$ and (b) fluorescence intensity around $350 \mathrm{~nm}$. The reaction was initiated by a $\mathrm{GdnHCl}$ concentration jump from $7 \mathrm{M}$ to $0.61 \mathrm{M}$. The curves were fitted to several exponentials. The inset in figure (b) shows the difference between the observed and fitted curves of the fluorescence measurement.

\subsection{Kinetic refolding reaction}

To investigate the kinetic refolding of canine milk lysozyme, we carried out stopped-flow experiments. The refolding reaction of lysozyme was induced by a concentration jump of $\mathrm{GdnHCl}$ from $7 \mathrm{M}$ to $0.61 \mathrm{M}$ by the stopped-flow mixing technique, and the resultant kinetics were observed by rapid CD measurement at $222 \mathrm{~nm}$ and fluorescence emission around $350 \mathrm{~nm}$. The refolding curve thus obtained is shown in Fig. 5. We assumed the signal of the refolded protein was identical to that of the $\mathrm{N}$ state. The observed kinetic curve was fitted by the non-linear least-squares method with the equation:

$$
A(t)=A_{0}+\sum_{i} A_{i} \exp \left(-k_{i} t\right)
$$

where $A(t)$ and $A_{0}$ are the signal values at time $t$ and the infinite time, respectively, and $A_{i}$ and $k_{i}$ are the amplitude and the apparent rate constant of phase $i$. The rate constants and relative amplitude we observed are shown in Table 2. In kinetic CD measurement, the ellipticity value extrapolated to zero time was much smaller than the value of the $\mathrm{U}$ state, suggesting that there must be a burst phase occurring within the dead time of the stopped-flow instrument $(25 \mathrm{~ms})$. Moreover, the overshoot of the ellipticity was observed. Therefore, the observed refolding curve is represented by a double exponential process. This suggests the existence of a kinetic folding intermediate after the burst-phase intermediate. In kinetic fluorescence measurement, there also exists a burst phase within the dead time of the measurement (4 ms). After the burst phase, the refolding curve is represented by four exponential terms. The rate constant of the fastest phase in the fluorescence measurement is comparable to that of the fast phase representing overshoot in the kinetic CD measurement. 
Table 2

Kinetic parameters of folding of canine milk lysozyme

\begin{tabular}{lccccc}
\hline CD at $222 \mathrm{~nm}$ & \multicolumn{5}{l}{ second } \\
\hline Phases & burst phase & first & & \\
\hline rate constant $\left(\mathrm{s}^{-1}\right)$ & & $14.6 \pm 2.0$ & $0.84 \pm 0.05$ & \\
relative amplitude $(\%)^{\mathrm{a}}$ & 99.0 & 9.6 & -8.6 & \\
\hline Fluorescence around 350 nm & & & & & \\
\hline Phases & burst phase & first & second & third & fourth \\
\hline rate constant $\left(\mathrm{s}^{-1}\right)$ & & $11.5 \pm 1.6$ & $0.99 \pm 0.02$ & $0.49 \pm 0.05$ & $0.071 \pm 0.006$ \\
relative amplitude $(\%)^{\mathrm{a}}$ & 54.4 & 0.8 & 29.4 & 9.3 & 6.1 \\
\hline
\end{tabular}

${ }^{\mathrm{a}}$ The signal change from the unfolded state to native state is defined as $100 \%$ of relative amplitude.

\section{Discussion}

\subsection{Comparison of the equilibrium unfolding transition between canine milk lysozyme and other proteins involved in lysozyme and $\alpha$-lactalbumin species}

The experimental data of the equilibrium unfolding of canine milk lysozyme in the present study are well represented by a three-state mechanism that involves only the $\mathrm{N}, \mathrm{I}$ and $\mathrm{U}$ states. The three-state mechanism is analogous to the three-state equilibrium unfolding of equine lysozyme and $\alpha$-lactalbumin induced by $\mathrm{GdnHCl}$ but apparently different from the unfolding mechanism of conventional non- $\mathrm{Ca}^{2+}$. binding lysozyme that is known to show a two-state unfolding without accumulation of the I state at equilibrium [10,15]. Consequently, canine milk lysozyme resembles equine lysozyme and $\alpha$-lactalbumin rather than conventional lysozyme in the unfolding behavior.

Although canine milk lysozyme represents the three-state unfolding transition as equine lysozyme and $\alpha$-lactalbumin do, there is a marked difference in the thermodynamic properties of the I state of canine milk lysozyme as compared with the properties of other lysozyme and $\alpha$-lactalbumin. The thermodynamic analysis of the GdnHCl-induced unfolding of holo canine milk lysozyme gave a $\Delta G_{\mathrm{IU}}^{\mathrm{H}_{2} \mathrm{O}}$ value of $6.20 \mathrm{kcal} \mathrm{mol}^{-1}$ and a $m_{\mathrm{IU}}$ value of $1.26 \mathrm{kcal} \mathrm{mol}^{-1} \mathrm{M}^{-1}$ (Figs 3, 4 and Table 1). Both of these values are significantly larger than the corresponding values of equine lysozyme $\left(\Delta G_{\mathrm{IU}}^{\mathrm{H}_{2} \mathrm{O}}\right.$ : $4.33 \mathrm{kcal} \mathrm{mol}^{-1}, m_{\mathrm{IU}}: 1.13 \mathrm{kcal} \mathrm{mol}^{-1} \mathrm{M}^{-1}$; [10]) and bovine $\alpha$-lactalbumin $\left(\Delta G_{\mathrm{IU}}^{\mathrm{H}_{2} \mathrm{O}}: 1.42 \mathrm{kcal} \mathrm{mol}^{-1}\right.$, $m_{\mathrm{IU}}: 0.75 \mathrm{kcal} \mathrm{mol}^{-1} \mathrm{M}^{-1}$; [15]) (Table 1), indicating that the I state of canine milk lysozyme is remarkably more stable than that of the other lysozyme and $\alpha$-lactalbumin.

Kobashigawa et al. (2000) [14] have also measured the GdnHCl-induced equilibrium unfolding curves of canine milk lysozyme by CD and fluorescence spectroscopy (Table 1). In contrast with the two separate transitions observed by CD at $295 \mathrm{~nm}$ as well as by fluorescence intensity around $350 \mathrm{~nm}$ in the present study (Figs 3(b) and 4(b)), the previous study has only reported a single cooperative transition that occurs below $3 \mathrm{M} \mathrm{GdnHCl}$. The present study clearly indicates that the three-state transition among N, I, and $\mathrm{U}$ well interprets the results of the simultaneous global fitting analysis of the different transition curves and that the second transition from I to $\mathrm{U}$ is accompanied by remarkable changes in the aromatic $\mathrm{CD}$ and the fluorescence intensity, suggesting that the I state has a rigid structure in which some aromatic side chains are tightly packed in asymmetric environment. Other differences between the present and the previous studies include the difference in the $\mathrm{pH}$ employed and the difference in the purification procedure of the protein; the previous study used $\mathrm{pH} 4.5$ instead of $\mathrm{pH} 7.0$, and did not adopt HPLC for the final purification. As compared with the thermodynamic parameters previously reported, $\Delta G_{\mathrm{NI}}^{\mathrm{H}_{2} \mathrm{O}}$ is 
significantly larger, and the difference in $\Delta G_{\mathrm{NI}}^{\mathrm{H}_{2} \mathrm{O}}$ between the present and the previous values is larger than the corresponding difference in $\Delta G_{\mathrm{IU}}^{\mathrm{H}_{2} \mathrm{O}}$. This indicates that the $\mathrm{N}$ state is more sensitive to the difference in $\mathrm{pH}$ than the I state. There are also other differences between the present and previously reported values, but considering the differences in the experimental conditions and procedure mentioned above, these might not be significant.

Recently, the study of the molten globule state has progressed extensively, and the difference of the state between $\alpha$-lactalbumin and lysozyme has been discussed in detail. The molten globule state of $\alpha$-lactalbumin has a nativelike tertiary fold, but it lacks tertiary packing interactions that are one of characteristics of native protein $[16,17]$. On the other hand, the molten globule state of equine lysozyme is stabilized by nativelike specific interaction. A hydrophobic cluster seems to be more densely packed in the state, and this may be responsible for the enhanced near-UV CD band $[10,18,19]$. Therefore, the molten globule state of canine milk lysozyme is more similar to that of equine lysozyme. In ${ }^{1} \mathrm{H}$ NMR spectrum measurements, however, stronger protection around the C- and D-helix of the aromatic cluster region is observed in the intermediates of equilibrium thermal unfolding, suggesting the existence of more specific packing interactions in the canine milk lysozyme molten globule [9]. This may be responsible for the extreme stability of the intermediate observed in the GdnHCl-induced equilibrium unfolding measurement of canine milk lysozyme.

\subsection{Kinetic refolding behavior of canine milk lysozyme}

The refolding curves of equine lysozyme and apo- $\alpha$-lactalbumin are known to be represented by a single exponential process after accumulation of a burst phase intermediate [10,20]. Although holo$\alpha$-lactalbumin exhibits biphasic refolding kinetics, this has been ascribed to the heterogeneity in the unfolded state [20]. Thus, there is only the burst phase intermediate between the native and fully unfolded states in equine lysozyme and $\alpha$-lactalbumin, and the burst phase intermediate is shown to be identical to the equilibrium MG state [8,10]. However, the refolding of canine milk lysozyme is represented by two or more exponential processes after accumulation of the burst phase intermediate. The results indicate that there are at least two intermediates, the burst-phase intermediate and the intermediate formed by the first phase of the exponential process, in the kinetic refolding of canine milk lysozyme. The burst-phase intermediate shows the native-like CD intensity in the peptide region and a 54\% change of the tryptophan fluorescence around $350 \mathrm{~nm}$ from the $\mathrm{U}$ to the $\mathrm{N}$ state. The intermediate formed by the first phase, however, exhibits the overshoot behavior in the time dependent ellipticity change at $222 \mathrm{~nm}$ and only a $1 \%$ change of the tryptophan fluorescence (Table 2). This suggests that the first phase of refolding may represent reorganization of the secondary structure within the already compact molten globule formed in the burst phase. The observation of the at least two kinetic intermediates would be related to the much higher stability of the equilibrium MG state in canine milk lysozyme. However, to determine which of these two kinetic intermediates corresponds to the equilibrium MG state, further studies are required.

\section{Conclusion}

We have shown that equilibrium unfolding of canine milk lysozyme is well represented by the threestate mechanism in which the N, I and U states are populated, and that the intermediate of the lysozyme is much more stable than that of equine lysozyme or $\alpha$-lactalbumin. We have also reported that there are at least two intermediates in the kinetic refolding of canine milk lysozyme: the burst-phase intermediate 
that have native-like CD intensity at $222 \mathrm{~nm}$ and some hydrophobic interactions, and the intermediate that is generated by reorganization of secondary structure. These characteristics we have observed are what make canine milk lysozyme distinctive among the lysozyme- $\alpha$-lactalbumin superfamily.

\section{References}

[1] K. Kuwajima, The molten globule state as a clue for understanding the folding and cooperativity of globular-protein structure, Proteins 6 (1989), 87-103.

[2] P.S. Kim and R.L. Baldwin, Intermediates in the folding reactions of small proteins, Annu. Rev. Biochem. 59 (1990), 631-660.

[3] M. Arai and K. Kuwajima, Role of the molten globule state in protein folding, Advan. Protein Chem. 53 (2000), $209-282$.

[4] K. Kuwajima, Y. Hiraoka, M. Ikeguchi and S. Sugai, Comparison of the transient folding intermediates in lysozyme and $\alpha$-lactalbumin, Biochemistry 24 (1985), 874-881.

[5] M. Ikeguchi, K. Kuwajima, M. Mitani and S. Sugai, Evidence for identity between the equilibrium unfolding intermediate and a transient folding intermediate: A comparative study of the folding reactions of $\alpha$-lactalbumin and lysozyme, Biochemistry 25 (1986), 6965-6972.

[6] K. Kuwajima, The molten globule state of $\alpha$-lactalbumin, FASEB J. 10 (1996), 102-109.

[7] O.B. Ptitsyn, How the molten globule became, Trends Biochem. Sci. 20 (1995), 376-379.

[8] M. Arai and K. Kuwajima, Rapid formation of a molten globule intermediate in refolding of $\alpha$-lactalbumin, Fold. Des. 1 (1996), 275-287.

[9] T. Koshiba, M. Yao, Y. Kobashigawa, M. Demura, A. Nakagawa, I. Tanaka, K. Kuwajima and K. Nitta, Structure and thermodynamics of the extraordinarily stable molten globule state of canine milk lysozyme, Biochemistry 39 (2000), 3248-3257.

[10] M. Mizuguchi, M. Arai, Y. Ke, K. Nitta and K. Kuwajima, Equilibrium and kinetics of the folding of equine lysozyme studied by circular dichroism spectroscopy, J. Mol. Biol. 283 (1998), 265-277.

[11] C.N. Pace, Determination and analysis of urea and guanidine hydrochloride denaturation curves, Meth. Enzymol. 131 (1986), 266-280.

[12] M. Kikuchi, K. Kawano and K. Nitta, Calcium-binding and structural stability of echidna and canine milk lysozymes, Protein Sci. 7 (1998), 2150-2155.

[13] T. Koshiba, T. Hayashi, I. Miwako, I. Kumagai, T. Ikura, K. Kawano, K. Nitta and K. Kuwajima, Expression of a synthetic gene encoding canine milk lysozyme in Escherichia coli and characterization of the expressed protein, Protein Eng. 12 (1999), 429-435.

[14] Y. Kobashigawa, M. Demura, T. Koshiba, Y. Kumaki, K. Kuwajima and K. Nitta, Hydrogen exchange study of canine milk lysozyme: Stabilization mechanism of the molten globule, Proteins 40 (2000), 579-589.

[15] M. Ikeguchi, K. Kuwajima and S. Sugai, $\mathrm{Ca}^{2+}$-induced alteration in the unfolding behavior of $\alpha$-lactalbumin, J. Biochem. (Tokyo) 99 (1986), 1191-1201.

[16] K. Kuwajima, K. Nitta, M. Yoneyama and S. Sugai, Three-state denaturation of $\alpha$-lactalbumin by guanidine hydrochloride, J. Mol. Biol. 106 (1976), 359-373.

[17] B.A. Schulman, C. Redfield, Z.Y. Peng, C.M. Dobson and P.S. Kim, Different subdomains are most protected from hydrogen exchange in the molten globule and native states of human $\alpha$-lactalbumin, J. Mol. Biol. 253 (1995), 651-657.

[18] L.A. Morozova, D.T. Haynie, C. Arico-Muendel, H. Van Dael and C.M. Dobson, Structural basis of the stability of a lysozyme molten globule, Nature Struct. Biol. 2 (1995), 871-875.

[19] L.A. Morozova-Roche, C.C. Arico-Muendel, D.T. Haynie, V.I. Emelyanenko, H. Van Dael and C.M. Dobson, Structural characterization and comparison of the native and A-states of equine lysozyme, J. Mol. Biol. 268 (1997), 903-921.

[20] K. Kuwajima, M. Mitani and S. Sugai, Characterization of the critical state in protein folding. Effects of guanidine hydrochloride and specific $\mathrm{Ca}^{2+}$ binding on the folding kinetics of $\alpha$-lactalbumin, J. Mol. Biol. 206 (1989), 547-561. 


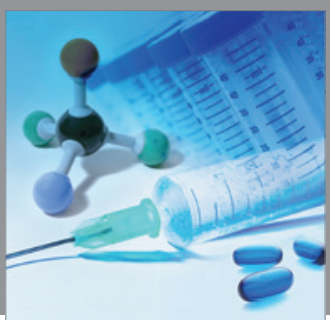

International Journal of

Medicinal Chemistry

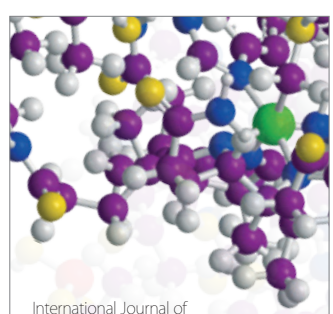

Carbohydrate Chemistry

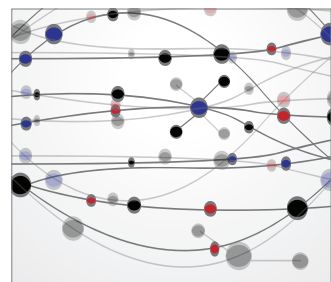

The Scientific World Journal
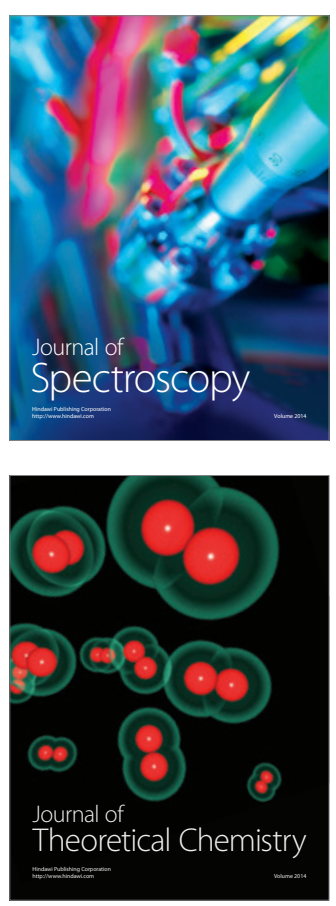
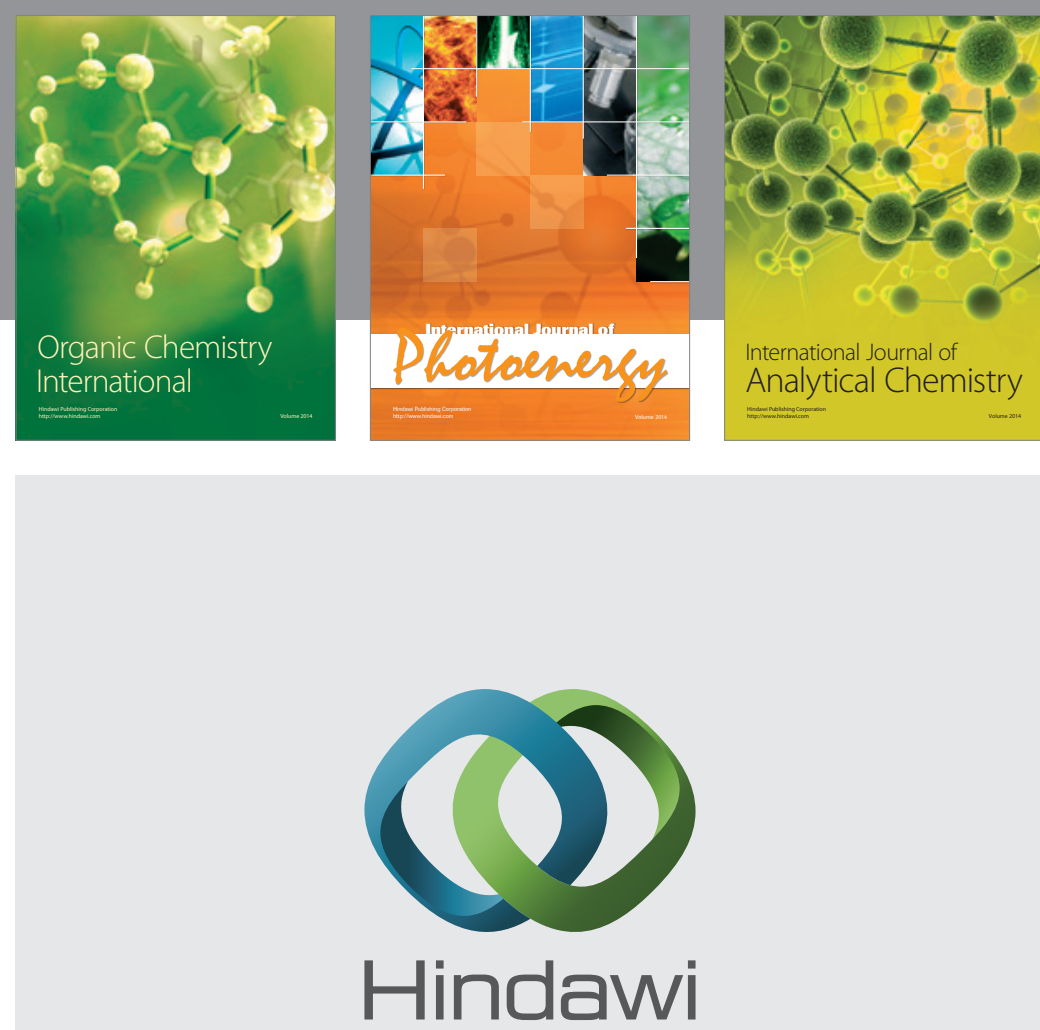

Submit your manuscripts at

http://www.hindawi.com
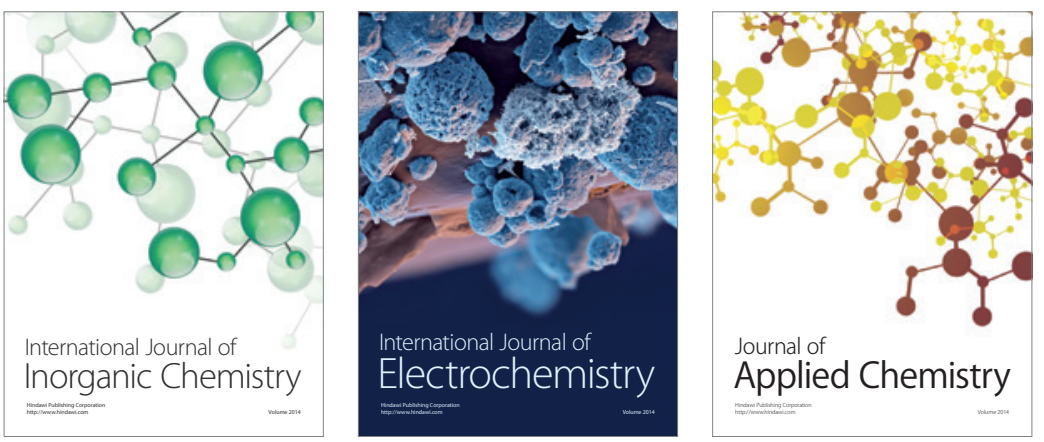

Journal of

Applied Chemistry
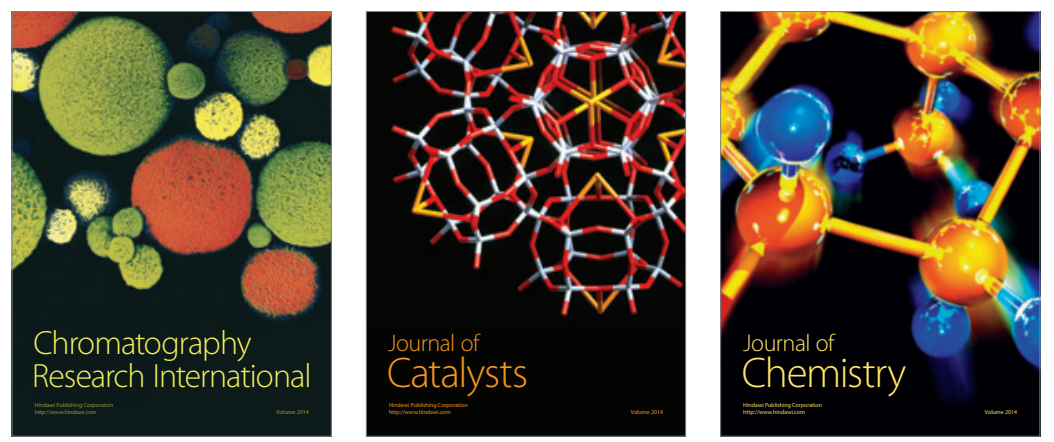
